\title{
Conductance and Shot Noise for Particles with Exclusion Statistics
}

\author{
Serguei B. Isakov ${ }^{\dagger}$, Thierry Martin ${ }^{\ddagger}$, and Stéphane Ouvry ${ }^{\dagger}$ \\ ${ }^{\dagger}$ Laboratoire de Physique Théorique et Modèles Statistiques, Bât. 100, Université Paris-Sud, 91405 Orsay Cedex, France \\ ${ }^{\ddagger}$ Centre de Physique Théorique, Université de Méditérranée P.O. Box 907, 13288 Marseille, France
}

(November 27, 1998)

\begin{abstract}
The first quantized Landauer approach to conductance and noise is generalized to particles obeying exclusion statistics. We derive an explicit formula for the crossover between the shot and thermal noise limits and argue that such a crossover can be used to determine experimentally whether charge carriers in FQHE devices obey exclusion statistics.
\end{abstract}

Recent experiments measuring quantum fluctuations in the tunneling current between two edges of a fractional Hall quantum liquid detected a fractional charge for the charge carriers [1,2]. At weak tunneling, quantum shot noise arises from the independent tunneling events of quasiparticles (Schottky limit): a noise measurement constitutes a direct measurement of the charge [1]. However, the implications of the statistics of the quasiparticles on the transport and noise have remained unclear. Laughlin's quasiparticles are supposed not only to carry fractional charge, but also to obey fractional statistics [3]. It was later argued that these quasiparticles might also be described [4] in terms of exclusion statistics [4, 5 . A description of edge excitations of fractional quantum Hall liquids in terms of Luttinger liquids allowed to make predictions on the transport and noise in integrable models where the tunneling is described by a scattering with point like impurities [6]. But it is not clear how to extract effects of statistics within those models.

Our purpose here is to study the implications of exclusion statistics of carriers on the quantum shot noise. To this aim, we use the Landauer approach [7] where effects of statistics are manifest. The reduction of the shot noise for fermions due to the Pauli exclusion principle predicted within this approach [8.9] was observed experimentally 10]. Also, differences between the fermion and boson noise have been pointed out [11]. In the fractional quantum Hall effect (FQHE) experiments [1, 2], a reduction of noise similar to that of fermions was observed.

In this Letter, we compute current and noise for exclusion statistics charge carriers in the first quantized version of the Landauer approach, the "wave packet" approach [9]. The resulting expressions are in agreement with the fluctuation dissipation theorem and exhibit quantum shot noise reduction. We propose that further experiments should look in more detail at the crossover regime to determine the statistics of the charge carriers.

Bose and Fermi current and noise. - Let us first consider for usual Bose or Fermi carriers the current and noise in a first quantized Landauer approach [9]. This is mandatory since in the case of exclusion statistics a second quantized formalism is not available at present.

In the Landauer setting, a current is transported be- tween a reservoir 1 on the left with chemical potential $\mu_{1}$ and a reservoir 2 on the right with chemical potential $\mu_{2}$ separated by a scattering region of arbitrary transmission $T$ (we assume $T$ not to depend on the energy of carriers). The probability to have $N$ bosons in a reservoir of chemical potential $\mu$ and in a quantum state of energy $\epsilon$ is $(1-x) x^{N}$, where $x \equiv e^{\beta(\mu-\epsilon)}$ is the Gibbs factor. The probability to have $N$ fermions is $(1+x)^{-1} x^{N}$ keeping in mind that, due to the Pauli principle, $N \geq 2$ is forbidden. Consider a scattering event where $N_{1}$ and $N_{2}$ particles, in a given quantum state of energy $\epsilon$, are incident from reservoirs 1 and 2 , respectively, and $k_{1}\left(k_{2}\right)$ of these incident particles are transmitted to reservoir 2 (1). The probability for such an event rewrites as (in the sequel the indices 1 and 2 always refer to reservoirs 1 and 2)

$$
\left(1 \mp x_{1}\right)^{ \pm 1} x_{1}^{N_{1}}\left(1 \mp x_{2}\right)^{ \pm 1} x_{2}^{N_{2}} \mathcal{W}_{k_{1} k_{2}}^{N_{1} N_{2}}
$$

where the upper and lower signs stand for bosons and fermions, respectively, and

$$
\mathcal{W}_{k_{1} k_{2}}^{N_{1} N_{2}} \equiv\left(\begin{array}{c}
N_{1} \\
k_{1}
\end{array}\right)\left(\begin{array}{c}
N_{2} \\
k_{2}
\end{array}\right) T^{k_{1}+k_{2}}(1-T)^{N_{1}+N_{2}-k_{1}-k_{2}}
$$

Since $\sum_{\substack{0 \leq k_{1} \leq N_{1} \\ 0 \leq k_{2} \leq N_{2}}} \mathcal{W}_{k_{1} k_{2}}^{N_{1} N_{2}}=1$, the probabilities (11) trivially sum to 1 when all possible scattering processes are taken into account, i.e. $N_{1}, N_{2}=0,1,2 \ldots$ in the Bose case and $N_{1}, N_{2}=0,1$ in the Fermi case.

Nothing has yet been said about the probability to have after scattering $N_{1}-k_{1}+k_{2}$ particles in reservoir 1 and $N_{2}-k_{2}+k_{1}$ particles in reservoir 2 . Still, scattering events with $N_{1}-k_{1}+k_{2} \geq 2$ or $N_{2}-k_{2}+k_{1} \geq 2$ should be strengthened for bosons because of their tendency to gather in the same state, whereas they should be forbidden for fermions because of the Pauli exclusion principle.

In the following, we will denote the Bose and Fermi summations as $\sum_{\substack{N_{1}=0 \\ N_{2}=0}}^{\infty} \sum_{\substack{0 \leq k_{1} \leq N_{1} \\ 0 \leq k_{2} \leq N_{2}}} \equiv \sum^{\infty}$ and $\sum_{N_{2}=0}^{N_{1}=0} \sum_{\substack{0 \leq k_{1} \leq N_{1} \\ 0 \leq k_{2} \leq N_{2}}} \equiv \sum^{1}$. The one dimensional line being oriented from the left to the right, the average algebraic number of carriers $\langle k\rangle \equiv\left\langle k_{1}-k_{2}\right\rangle$ of charge $q$ (which may be different from the electron charge) at energy $\epsilon$ transmitted through the scattering region - related to the average $1 \mathrm{~d}$ current by $\langle I\rangle=(q / h) \int_{0}^{\infty}\langle k\rangle d \epsilon$ - is 


$$
\langle k\rangle=\sum^{\infty, 1}\left(1 \mp x_{1}\right)^{ \pm 1} x_{1}^{N_{1}}\left(1 \mp x_{2}\right)^{ \pm 1} x_{2}^{N_{2}} \mathcal{W}_{k_{1} k_{2}}^{N_{1} N_{2}}\left(k_{1}-k_{2}\right)
$$

leading to the Landauer formula $\langle k\rangle=T\left(f_{1}-f_{2}\right)$ where $f(x)=x /(1 \mp x)$ is the mean ocupation number at energy $\epsilon$ in the Bose and Fermi cases. Can the spectral noise be obtained in this way? The average square of the number of transmitted particles is

$\left\langle k^{2}\right\rangle=\sum^{\infty, 1}\left(1 \mp x_{1}\right)^{ \pm 1} x_{1}^{N_{1}}\left(1 \mp x_{2}\right)^{ \pm 1} x_{2}^{N_{2}} \mathcal{W}_{k_{1} k_{2}}^{N_{1} N_{2}}\left(k_{1}-k_{2}\right)^{2}$

to yield the fluctuations - related to the zero frequency spectral density of noise by $P=\frac{2 q^{2}}{h} \int_{0}^{\infty}\left(\left\langle k^{2}\right\rangle-\langle k\rangle^{2}\right) d \epsilon-$

$$
\left\langle k^{2}\right\rangle-\langle k\rangle^{2}=T\left(f_{1}+f_{2}\right) \pm T^{2}\left(f_{1}^{2}+f_{2}^{2}\right) .
$$

However, one has to add to (3) the term $\pm 2 T(1-T) f_{1} f_{2}$ to recover the standard result obtained from second quantization [1]

$$
\begin{aligned}
\left\langle k^{2}\right\rangle-\langle k\rangle^{2}= & T f_{1}\left(1 \pm f_{1}\right)+T f_{2}\left(1 \pm f_{2}\right) \\
& \mp T(1-T)\left(f_{1}-f_{2}\right)^{2} .
\end{aligned}
$$

It is straightforward to understand, in the Fermi case, the origin of $2 T(1-T) f_{1} f_{2}$ which has to be substracted from (3) to get (14): this term is precisely built by the two scattering processes $N_{1}=N_{2}=k_{2}=1, k_{1}=0$ and $N_{1}=$ $k_{1}=N_{2}=1, k_{2}=0$, with respectively $N_{1}-k_{1}+k_{2}=2$ particles in reservoir 1 and $N_{2}-k_{2}+k_{1}=2$ particles in reservoir 2 after scattering. Precisely, both these processes should be suppressed because of the Pauli exclusion principle. Why the same term has to be added in the Bose case to take into account the strenghtening of scattering processes where several particles occupy after scattering the same energy state in reservoir 1 or 2 has yet to be understood in this first quantized approach.

It is well known that the second quantized spectral noise defined from (4) satisfies two independent constraints:

(i) Fluctuation-dissipation theorem: when $\mu_{1}=\mu_{2}$, the noise is proportional to the conductance, which in particular implies that the $T^{2}$ term has to vanish when the chemical potentials are equal (thermal noise).

(ii) Quantum suppression of the noise at zero temperature: the noise scales as $T(1-T)$ at $\beta=\infty$ (shot noise).

Exclusion statistics. - Let us generalize the first quantized approach to $1 \mathrm{~d}$ exclusion statistics [5] particles with statistics parameter $g$. For exclusion statistics, the one quantum state grand-partition function $\Xi$ at energy $\epsilon$ and chemical potential $\mu$ satisfies the transcendental equation $\Xi-x \Xi^{1-g}=1$. The mean occupation number $f(x) \equiv x \frac{\Xi^{\prime}}{\Xi}$ (the prime means derivative with respect to $x$ ) satisfies

$$
x=\frac{f}{(1-g f)^{g}[1+(1-g) f]^{1-g}} .
$$

It interpolates between the usual Bose and Fermi distributions when $g$ varies from 0 to 1 .
¿From $\Xi-x \Xi^{1-g}=1$, one has $\Xi=\sum_{N=0}^{\infty} x^{N} P_{N}$ where $P_{N} \equiv \prod_{k=2}^{N} \frac{k-g N}{k}$. The probability to have $N$ exclusion statistics particles in a reservoir of chemical potential $\mu$ and in a quantum state of energy $\epsilon$ should thus be

$$
\frac{1}{\sum_{N=0}^{\infty} x^{N} P_{N}} x^{N} P_{N} .
$$

Eq. (6) indeed reproduces the Bose (Fermi) probabilities when $g=0(g=1)$, but it can be negative for certain values of $g \in] 0,1[$. This should not be considered as problematic since exclusion statistics has to be understood for a macroscopic ensemble of interacting particles occupying a macroscopic number of quantum states. In other words, one quantum state probability or one quantum state grand partition function, if precisely defined formally, have no real physical meaning, as well as any spectral quantity computed from them. However, they become physically relevant when integrated over the whole range of the energy spectrum, as we will see later.

Let us now follow the first quantized procedure used above in the Bose and Fermi cases. The probabilities (1) should now become

$$
\frac{x_{1}^{N_{1}} P_{N_{1}}}{\Xi_{1}} \frac{x_{2}^{N_{2}} P_{N_{2}}}{\Xi_{2}} \mathcal{W}_{k_{1} k_{2}}^{N_{1} N_{2}}
$$

Again, one has $\sum^{\infty} \frac{x_{1}^{N_{1}} P_{N_{1}}}{\Xi_{1}} \frac{x_{2}^{N_{2}} P_{N_{2}}}{\Xi_{2}} \mathcal{W}_{k_{1} k_{2}}^{N_{1} N_{2}}=1$. The average number of transmitted carriers is

$$
\begin{aligned}
\langle k\rangle & =\sum^{\infty} \frac{x_{1}^{N_{1}} P_{N_{1}}}{\Xi_{1}} \frac{x_{2}^{N_{2}} P_{N_{2}}}{\Xi_{2}} \mathcal{W}_{k_{1} k_{2}}^{N_{1} N_{2}}\left(k_{1}-k_{2}\right) \\
& =T\left(f_{1}-f_{2}\right)
\end{aligned}
$$

i.e. the finite temperature Landauer formula also holds for exclusion statistics. The $1 \mathrm{~d}$ average current then reads

$$
\langle I\rangle \equiv \frac{q}{h} \int_{0}^{\infty} T\left(f_{1}-f_{2}\right) d \epsilon .
$$

By setting the voltage as $q V \equiv \mu_{1}-\mu_{2}$, the linear conductance follows as

$$
G \equiv \lim _{V \rightarrow 0} \frac{\langle I\rangle}{V}=\frac{q^{2}}{h} T f_{1}(\epsilon=0),
$$

where $f_{1}(\epsilon=0)$ is the mean occupation number at zero energy and chemical potential $\mu_{1}\left(f_{1}(\epsilon=\infty)=0\right)$.

Similarly, the spectral noise should be defined from

$$
\left\langle k^{2}\right\rangle=\sum^{\infty} \frac{x_{1}^{N_{1}} P_{N_{1}}}{\Xi_{1}} \frac{x_{2}^{N_{2}} P_{N_{2}}}{\Xi_{2}} \mathcal{W}_{k_{1} k_{2}}^{N_{1} N_{2}}\left(k_{1}-k_{2}\right)^{2},
$$

which yields

$$
\begin{aligned}
\left\langle k^{2}\right\rangle-\langle k\rangle^{2}= & T\left(f_{1}+f_{2}\right) \\
& +T^{2}\left[f_{1}\left(C_{1}-1\right)+f_{2}\left(C_{2}-1\right)\right],
\end{aligned}
$$


where $C(x) \equiv x \frac{f^{\prime}}{f}=(1-g f)[1+(1-g) f]$ is related 112 to the fluctuation of the mean occupation number $\left\langle\delta f^{2}\right\rangle=f C$. Again, one is confronted to the same difficulty as in the Bose and Fermi cases, that is to say a term has to be added to (11) to ensure that both the fluctuation-dissipation theorem and quantum suppression at zero temperature hold. We believe that the fluctuation-dissipation theorem is valid for exclusion statistics, eventhough there is no definite proof that it is indeed the case. The fluctuation-dissipation theorem requires that when $\mu_{1}=\mu_{2}$, the $T^{2}$ part of the noise should vanish. In the Bose and Fermi cases, the term added to (3) is proportional to $T(1-T)$ and symmetric in 1 and 2. Let us generalize this construction to exclusion statistics by adding to the first quantized spectral noise (11) a term proportional to $T(1-T)$, symmetric in 1 and 2 , and such that the $T^{2}$ part disappears when $\mu_{1}=\mu_{2}$. These constraints uniquely determine this term to be $T(1-T)\left[f_{1}\left(C_{2}-1\right)+f_{2}\left(C_{1}-1\right)\right]$ which results in

$$
\begin{aligned}
\left\langle k^{2}\right\rangle-\langle k\rangle^{2}= & T\left(f_{1} C_{1}+f_{2} C_{2}\right) \\
& -T(1-T)\left(f_{1}-f_{2}\right)\left(C_{1}-C_{2}\right),
\end{aligned}
$$

reproducing the Bose (Fermi) cases (4) when $g=0$ $(g=1)$. The zero frequency spectral density of noise follows as

$$
\begin{aligned}
P=2 \frac{q^{2}}{h} \int_{0}^{\infty} & \left(T\left(f_{1} C_{1}+f_{2} C_{2}\right)\right. \\
& \left.-T(1-T)\left(f_{1}-f_{2}\right)\left(C_{1}-C_{2}\right)\right) d \epsilon .
\end{aligned}
$$

Does it satifies the fluctuation-dissipation theorem and quantum suppression?

(i) Fluctuation-dissipation theorem: (13) yields when $\mu_{1}=\mu_{2}$ the thermal noise

$$
P_{\text {thermal }}=2 \frac{q^{2}}{h} 2 T \int_{0}^{\infty} f_{1} C_{1} d \epsilon=2 \frac{q^{2}}{h} \frac{2 T}{\beta} f_{1}(\epsilon=0) .
$$

Thus, from (10), the relation $P_{\text {thermal }}=4 G / \beta$ holds for exclusion statistics, which manifests the fluctuationdissipation theorem.

(ii) Quantum suppression of the shot noise: at zero temperature on the other hand, only the $T(1-T)$ term contributes to the noise (13). Since at zero temperature

$$
\int_{0}^{\infty}\left(f_{1}-f_{2}\right)\left(C_{1}-C_{2}\right) d \epsilon=-\frac{1}{g}\left(\mu_{1}-\mu_{2}\right),
$$

one obtains the shot noise

$$
P_{\text {shot }}=2 \frac{q^{2}}{h} \frac{1}{g} T(1-T)\left(\mu_{1}-\mu_{2}\right)
$$

which is indeed proportional to $T(1-T)$. Note also that when $g \rightarrow 0$, (15,16) become meaningless, a mere reflection of the absence of a well defined Bose spectral noise at small temperature.
Crossover from thermal to shot noise. - Let us now assume that $e^{\beta \mu_{1}} \simeq e^{\beta \mu_{2}} \gg 1$, even though $\beta\left(\mu_{1}-\mu_{2}\right)$ can be small (thermal noise) or large (shot noise) [13]. The question we now ask is what is the crossover regime which interpolates between these two situations?

When $e^{\beta \mu_{1}}, e^{\beta \mu_{2}} \gg 1$, one has that not only $f(\epsilon=$ $0)=1 / g$ but also $\int_{0}^{\infty} f d \epsilon=\mu / g$. Then in the shot noise limit the current (9) and the conductance (10) become

$$
\langle I\rangle=\frac{q}{h} T \frac{\mu_{1}-\mu_{2}}{g}, \quad G=\frac{q^{2}}{h} \frac{1}{g} T .
$$

The conductance in (17) is a generalization to exclusion statistics of the usual Landauer conductance. Comparing (16) and (17), one has

$$
P_{\text {shot }}=2 q\langle I\rangle(1-T) .
$$

We stress that the shot noise (18) has a universal form independent of the statistics of charge carriers. When $T \rightarrow 0$, the Schottky limit $P_{\text {shot }}=2 q\langle I\rangle$ is obtained. It was used to measure the fractional charge $q=e / 3$ of the carriers in $\nu=1 / 3$ FQHE devices [1]. Remarkably, the quantum suppression factor $1-T$ was also confirmed experimentally when $T$ is not small [1] for charge carriers which are supposed to obey fractional or exclusion statistics. Note that the average current $\langle I\rangle$ transmitted through the scattering region should be identified with the tunneling (or backscattering) current in the experiments [1.2].

If we assume that the charge carriers are $1 \mathrm{~d}$ quasiholes obeying exclusion statistics and carrying a charge $q=g e$ then the conductance in (17) becomes $G=\frac{e^{2}}{h} g T$, the FQHE conductance for the tunneling current.

Keeping $e^{\beta \mu_{1}}, e^{\beta \mu_{2}} \gg 1$, the energy integration of the spectral noise (13) can be simplified to

$$
P=\frac{2 q^{2}}{h} \frac{1}{g} \frac{2}{\beta}[T+T(1-T) \delta P],
$$

where the dimensionless excess noise

$$
\delta P=g \int_{0}^{\infty} \frac{\left[f\left(a^{2} x\right)+f\left(a^{-2} x\right)\right] C(x)}{2 x} d x-1,
$$

which only depends on the parameter $a \equiv \exp (\beta q V / 2)$, represents the noise in units of $4 G(1-T) / \beta$. Note that $\delta P \rightarrow 0$ when $\beta q V / 2 \rightarrow 0$ (thermal noise), and $\delta P \rightarrow \beta q V / 2$ when $\beta q V / 2 \rightarrow \infty$ (shot noise), as it should.

In the Fermi case $g=1$, with $q=e$, (19,20) yield the standard result

$P=\frac{2 e^{2}}{h} \frac{2}{\beta}\left[T+T(1-T)\left(\frac{\beta e V}{2} \operatorname{coth} \frac{\beta e V}{2}-1\right)\right]$.

In the case $g=1 / 2$, where $f(x)=x / \sqrt{1+x^{2} / 4}$, one obtains if $a<1$ 
$\delta P=\frac{\arctan \sqrt{a^{-4}-1}}{2 \sqrt{a^{-4}-1}}-\frac{1}{4 \sqrt{1-a^{4}}} \ln \frac{1-\sqrt{1-a^{4}}}{1+\sqrt{1-a^{4}}}-1$,

and if $a>1, a \rightarrow 1 / a$ in the above expression. In the case of interest $g=1 / 3$, i.e. fractional statistics quasihole in FQHE devices at filling factor $\nu=1 / 3$, one has

$f(x)=\frac{3}{2}\left(w+\frac{y}{w}\right), w^{3} \equiv y(1+\sqrt{1-y}), y \equiv \frac{\frac{4}{27} x^{3}}{1+\frac{4}{27} x^{3}}$.

The thermal and shot noise rewrite respectively as

$$
P_{\text {thermal }}=\frac{4}{3} \frac{e^{2}}{h} T \frac{1}{\beta}, \quad P_{\text {shot }}=\frac{2}{3} \frac{e^{2}}{h} T(1-T) \frac{e V}{3} .
$$

The crossover has been studied experimentally [1, 2] at $\nu=1 / 3$. In [2], by replacing in the fermion result (21), the quantum of conductance $e^{2} / h \rightarrow e^{2} /(3 h)$ and $\beta e V \rightarrow \beta e V / 3$, a reasonably good agreement with the experimental data could be obtained. These two changes happen to be fine tuned such that the thermal and shot noises coincide with (22). We propose here to go beyond this heuristic approach and compare the experimental data with the exact crossover formula (19,20) when $g=1 / 3$ and $q=e / 3$. The dimensionless excess noise (20), being a function of a single variable $\beta q V / 2$, can easily be analyzed numerically. In principle, the crossover from the shot to thermal noise can be achieved in two ways: either by varying the voltage $V$ at fixed temperature (the standard experimental setting to look at the crossover as a function of the backscattering current [2]), or vice versa. In the former case, the dimensionless excess noise (20) is well suited. Note in particular the asymptotic behavior when $\beta q V / 2 \rightarrow \infty$ (shot noise) $\delta P-\frac{\beta q V}{2}=c(g)-1+\ldots$ where $c(g)$ depends only on $g$ and the dots represent terms vanishing as $\beta q V / 2 \rightarrow \infty$. One has $c(1)=0, c(1 / 2)=\ln 2$ and we found numerically that $c(1 / 3) \approx 0.5493$ from which one can infer that $c(1 / 3)=(\ln 3) / 2$. In the shot noise limit, this subleading constant term contains information about the statistics of the carriers.

When the crossover is achieved by varying the temperature at a fixed voltage, the dimensionless excess noise $\delta P^{\prime}=(2 / \beta q V) \delta P$, which counts the noise in units of $2 G(1-T) q V$, is more appropriate. It is displayed in Fig. 1 for $g=1 / 3$.

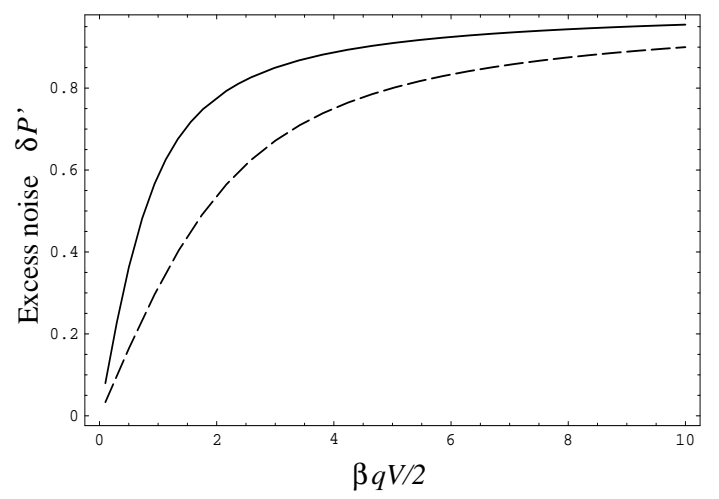

FIG. 1. The dimensionless crossover function $\delta P^{\prime}$ for $g=1 / 3$ and $q=e / 3$ representing the excess noise in units of $2\left(e^{2} / 3 h\right) T(1-T) e V / 3$ as a function of $\beta q V / 2$ (solid line). The same for the fermionic excess noise with $e^{2} / h \rightarrow e^{2} /(3 h)$ and $\beta e V \rightarrow \beta e V / 3$ as in [2] (dashed line).

The difference between the two curves is maximal for $\beta q V / 2 \simeq 1$ and vanishes when $\beta q V / 2 \rightarrow 0, \infty$.

As to tunneling experiments in FQHE devices, the thermal noise limit is eventually fixed by the fluctuation dissipation theorem $P_{\text {thermal }}=4 G / \beta$, where $G=\nu e^{2} / h$ is the FQHE conductance. On the other hand, the quantum shot noise has the universal form (18) independent of the statistics of the charge carriers. The statistics of charge carriers can be experimentally determined by analyzing the crossover between these two limits. This should in particular allow for an unambiguous experimental discrimination between fractional charge carriers with Fermi statistics and fractional charge carriers with exclusion statistics 14].

We would like to thank M. Büttiker and C. Glattli for discussions.

[1] L. Saminadayar, D. C. Glattli, Y. Jin, and B. Etienne, Phys. Rev. Lett. 79, 2526 (1997).

[2] R. de-Picciotto et al., Nature 389, 162 (1997).

[3] H. Störmer, D. C. Tsui and A. Gossard, Phys. Rev. Lett. 48, 1559 (1982); R. B. Laughlin, Phys. Rev. Lett. 50, 1395 (1983).

[4] F. D. M. Haldane, Phys. Rev. Lett. 67, 937 (1991).

[5] S. B. Isakov, Mod. Phys. Lett. B 8, 319 (1994); Int. J. Mod. Phys. A 9, 2563 (1994); A. Dasnières de Veigy and S. Ouvry, Phys. Rev. Lett. 72, 600 (1994); Mod. Phys. Lett. A 10, 1 (1995); Y.-S. Wu, Phys. Rev. Lett. 73, 922 (1994).

[6] C. L. Kane and M. P. A. Fisher, Phys. Rev. Lett. 72, 724 (1994); C. de C. Chamon, D. E. Freed, and X. G. Wen, Phys. Rev. B 51, 2363 (1995); P. Fendley, A. W. W. Ludwig, and H. Saleur, Phys. Rev. Lett. 75, 2196 (1995); P. Fendley and H. Saleur, Phys. Rev. B 54, 10845 (1996).

[7] R. Landauer, Philos. Mag. 21, 863 (1970); Y. Imry "Introduction to Mesoscopic Physics" (Oxford, 1997).

[8] G. Lesovik, JETP Lett. 49, 593 (1989); M. Büttiker, Phys. Rev. Lett. 65, 2901 (1990)

[9] T. Martin and R. Landauer, Phys. Rev. B 45, 1742 (1992).

[10] M. Reznikov et al., Phys. Rev. Lett. 75, 3340 (1995); A. Kumar et al., Phys. Rev. Lett. 76, 2778 (1996).

[11] M. Büttiker, Phys. Rev. B 46, 12485 (1992).

[12] A. K. Rajakopal, Phys. Rev. Lett. 74, 1048 (1995).

[13] The experiments [1]2] are performed at low temperature, of the order of $10-150 \mathrm{mK}$.

[14] We note that (9), (10), and (13) do not rely on the specific form of the exclusion statistics distributions (5) and are in fact valid for generic distributions; for example, for those proposed recently for edge quasiparticles in non-abelian FQHE states, see K. Schoutens, Phys. Rev. Lett. 81, 1929 (1998). 